\title{
ORIGINAL 2017: Rehabilitación Pulmonar en el Adulto Mayor con Neumopatía Crónica Obstructiva y/o Interstical en el Hospital Nacional de Geriatría y Gerontología, Dr. Raúl Blanco Cervantes de Mayo del 2012 a Junio del 2013
}
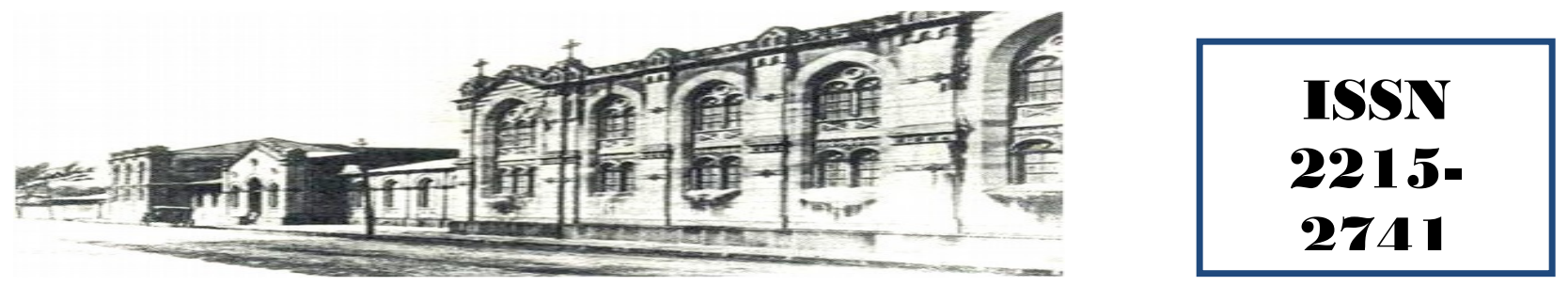

Haspital San quan de Dios. San José. Costa Rica. Fundado en 1845

Recibido:

$10 / 09 / 2017$

Aceptado:

$15 / 10 / 2017$

Karla Araya Aquilar ${ }^{1}$

1 Médico especialista en Geriatría y Gerontología, Universidad de Costa Rica. Hospital Nacional de Geriatría y Gerontología, Dr. Raúl Blanco Cervantes. Correo Electrónico: karlaarayaa@gmail.com

\section{RESUMEN}

El objetivo principal del estudio es describir la respuesta de los adultos mayores con neumopatía obstructiva $\mathrm{y} / \mathrm{o}$ intersticial a un programa de rehabilitación pulmonar en sus diferentes componentes. Se define rehabilitación pulmonar como un programa de ejercicios de entrenamiento y al menos uno de los siguientes componentes: educación, apoyo psicosocial y nutricional, como ha sido definido en otros estudios.

Métodos: Estudio observacional, descriptivo y retrospectivo.

Resultados: De los 12 pacientes, solo nueve finalizaron por completo el programa. La disnea, según la escala del mMRC, mejoró en el $42 \%$ y se mantuvo sin cambios en el 58\%. Hubo mejoría significativa en la caminata de seis minutos $(\mathrm{p}=0,009)$, en la PIMáx en el $83 \%$, con un promedio de $16,6 \mathrm{kPa}(16,9 \mathrm{cmH} 2 \mathrm{O})$; el índice de BODE en el $100 \%$ de los pacientes $(\mathrm{p}=0,015)$. En éste índice, la mayor mejoría se dio en los componentes de disnea $(p=0,007)$ y la C6M ( $\mathrm{p}=0,009)$. En el cuestionario de Saint George, el $75 \%$ obtuvo una respuesta positiva al programa, con mayor respuesta en los síntomas. No hubo mejoría en el FEV1 o FEV1/FVC. La resistencia muscular de extremidades superiores e inferiores aumentó.

Ninguna variable tuvo correlación significativa con edad, sexo y FEV1.

Conclusiones: La rehabilitación pulmonar en personas adultas mayores con neumopatía obstructiva $\mathrm{y} / \mathrm{o}$ intersticial evidencia una respuesta estadísticamente significativa en la caminata de $\mathrm{C} 6 \mathrm{M}$, índice de BODE, fuerza 
muscular de músculo respiratorios y resistencia muscular en extremidades superiores e inferiores. Con mejoría, pero sin significancia estadística en la escala de disnea del mMRC, y cuestionario de Saint George.

No se obtuvieron cambios en los parámetros espirométricos.

\section{PALABRAS CLAVE}

Rehabilitación pulmonar; enfermedad pulmonar crónica; capacidad de ejercicio; calidad de vida relacionada a la salud.

\section{ABSTRACT \\ The main objective of the study is to describe the response of the elderly patient with pulmonary osbtructive or interstitital lung disease to pul- monary rehabilitation program. Pulmonary reha- bilitation is defined as a training exercise pro- gram and at least one of the following compo- nents: education, psychosocial and nutritional support, as defined in other studies.}

Methods: Observational, descriptive and retrospective study.

Results: Of the 12 patients, only 9 completed the program. Dyspnea, according to the MMRC scale, improved by $42 \%$ and remained unchanged at 58\%. There was a significant improvement in the six-minute walk $(\mathrm{p}=0.009)$, in the PIMax in $83 \%$, with an average of $16.6 \mathrm{kPa}$ (16.9 $\mathrm{cmH} 2 \mathrm{O}$ ); the BODE index in $100 \%$ of patients $(p=0.015)$. In this index, the greatest improvement occurred in dyspnea components $(\mathrm{p}=$ $0.007)$ and W6M ( $\mathrm{p}=0.009)$. In the Saint George questionnaire, $75 \%$ received a positive response, with a greater response in symptoms. There was no improvement in FEV1 or FEV1 / FVC. Muscle endurance of upper and lower extremities increased. No variable had significant correlation with age, sex and FEV1.

Conclusions: Pulmonary rehabilitation in older adults with pulmonary osbtructive or interstitital lung disease shows a statistically significant response in the C6M walk, BODE index, respiratory muscle muscle strength and muscular endurance in upper and lower extremities. With im- provement, but not statistically significant in the mMRC dyspnea scale, and Saint George questionnaire.

There were no changes in the spirometric parameters

\section{KEY WORDS}

Pulmonary rehabilitation; Chronic lung disease; Exercise capacity; Quality of life related to health.

\section{INTRODUCCIÓN}

En las últimas décadas, se ha experimentado un envejecimiento de la población, a nivel mundial y nacional. La esperanza de vida en Costa Rica aumentó de 46,9 años en 1930 a 79,2 años actualmente ${ }^{(1)}$

Según las proyecciones de población vigentes, para el 2008 había aproximadamente $278 \mathrm{mil}$ personas adultas mayores en Costa Rica, constituyendo el $6 \%$ de la población. Este porcentaje de población de 65 años y más continúa en incremento, proyectando que para el 2050 sean $1.262 .311^{(1)}$.

Estos cambios poblacionales obligan a mejorar el conocimiento del envejecimiento normal $\mathrm{y}$ patológico.

El envejecimiento de la estructura pulmonar condiciona a alteraciones de su funcionalidad y pueden repercutir en enfermedades crónicas pulmonares y calidad de vida del adulto mayor.

El consumo de tabaco ha aumentado, llevando a un ascenso en la prevalencia de enfermedades pulmonares, dentro de éstas, la enfermedad pulmonar obstructiva crónica, la más frecuente. Se considera actualmente la tercera causa de muerte en los Estados Unidos (2), la cuarta en Costa Rica (Ministerio de Salud Costa Rica, 2011), y la doceava causa de discapacidad ${ }^{(3)}$. Las enfermedades pulmonares aumentan la mortalidad y morbilidad en la población general, y especialmente en los adultos mayores.

Las personas con patología pulmonar obstructiva y/o intersticial, con frecuencia desarrollan disnea progresiva de esfuerzo, afectación de la calidad de vida relacionada con la salud (CVRS), y limitación variable de su capacidad para realizar actividades cotidianas. Como consecuencia, se ven forzados a optar por un estilo de vida sedentario ${ }^{(3)}$. 
La rehabilitación pulmonar es una intervención multidisciplinaria y global, que ha demostrado ser eficaz desde la perspectiva de la medicina basada en la evidencia para los pacientes con enfermedades respiratorias crónicas, que a menudo han disminuido las actividades de la vida diaria. Ésta debe formar parte de un tratamiento individualizado del paciente, dirigido a reducir los síntomas, optimizar la capacidad funcional, incrementar la participación y reducir los costes sanitarios a través de la estabilización o reversión de las manifestaciones sistémicas de la enfermedad ${ }^{(4)}$.

Durante las últimas décadas se ha visto un progresivo desarrollo de la rehabilitación respiratoria como forma de tratamiento de la EPOC y otras patologías pulmonares crónicas de tipo restrictivo. Se ha demostrado que la limitación al ejercicio evidenciada por los pacientes con enfermedades obstructivas y restrictivas, viene determinada no sólo por su alteración ventilatoria, sino también por la presencia de una disfunción de los músculos esqueléticos. El entrenamiento muscular es capaz de mejorar la capacidad oxidativa muscular e incrementar su capacidad de ejercicio. Por otra parte, el objetivo actual del tratamiento de una enfermedad crónica no se limita a mejorar la supervivencia de la enfermedad, sino que intenta disminuir el impacto negativo que ésta tiene en la calidad de vida de los pacientes.

Existen estudios que demuestran que en los pacientes con enfermedades pulmonares, su calidad de vida se relaciona más con la sintomatología de disnea y con la tolerancia al ejercicio que con la alteración ventilatoria. Actualmente existe evidencia de que la rehabilitación respiratoria mejora la tolerancia al ejercicio y la calidad de vida de los pacientes con EPOC, asma y enfermedades de tipo restrictivo, aunque todavía no existen suficientes estudios que demuestren su repercusión en la supervivencia de los enfermos ${ }^{(5)}$.

\section{DISCUSIÓN}

La EPOC fue la patología más frecuente, con predominio en estadio severo, seguido por asma y EPID. En Estados Unidos, en el National Health and Nutrition Examination Survey (NHANES) III, se estima la prevalencia de la EPOC de 1988 a 1994, con $14,3 \%$ de la población adulta con limitación al flujo aéreo por espirometría, de los cuales $1,5 \%$ posee enfermedad moderada a severa con FEV1 menor a 50\%. Además, reporta aumento de su prevalencia conforme aumenta la edad, es decir, es mayor entre los 65 a 85 años ${ }^{(3)}$.

La verdadera prevalencia del asma en las personas adultas mayores es subdiagnósticada o se asocia el síndrome de traslape Asma - EPOC. Algunos estudios sugieren la prevalencia entre $6,5 \%$ y $17 \%{ }^{(10)}$. Otros estudios realizados en Estados Unidos y Europa reportan que de un 6 a un $8,5 \%$ de la población mayor de 60 años posee asma, con un inicio de la enfermedad antes de los $40^{(11)}$. La alta prevalencia en nuestro estudio se justifica por ser una muestra tan pequeña y el ingreso directo del servicio de neumología.

En cuanto a las enfermedades del parénquima pulmonar o intersticiales, en Estados Unidos se ha estimado una prevalencia de 20 a 40 por cada 100000 admisiones hospitalarias por año. La fibrosis pulmonar idiopática es la más común, con una prevalencia de 6 a 14,6 por cada 100000 personas en diferentes series. En mayores de 75 años puede aumentar hasta 175 por cada 100000 (3).

Las dificultades de movilidad y funcionalidad básica e instrumental son más frecuentes en las mujeres y se incrementan con la edad ${ }^{(1)}$. Este patrón se respeta en el estudio, pese a ser una muestra muy pequeña y estar ausentes los datos en el $41,7 \%$.

El alto de grado de independencia en ABVD y con un compromiso leve en la AIVD, era de esperar por el tipo de programa implementado.

En el I Informe Estado de Situación de la Persona Adulta Mayor en Costa Rica, se reporta la prevalencia de deterioro cognitivo severo hasta en un $14 \%$, sin especificar los otros estadios de déficit cognitivo. La incidencia de Enfermedad de Alzheimer está relacionada a la edad, y se duplica cada cinco años después de los 65 , con una prevalencia de $30 \%$ o más en mayores de 85 años ${ }^{(12)}$. Un estudio publicado en Mayo Clinic Proceedings, donde se estudiaron alrededor de 2000 personas entre 70 a 89 años. De ellos, 1600 tenían cognición normal, 317 DCL, y alrededor de 288 padecían de EPOC. Se descubrió una relación entre EPOC y una probabilidad doblemente mayor de presentar 
DCL, la cual empeoraba al aumentar los años de padecer la neumopatía ${ }^{(10)}$.

El reporte del estado cognitivo en la muestra estudiada se realizó por medio del MMSE y test del reloj, sólo una persona presentó resultados anormales, lo que podría corresponder a DCL. Tales resultados no concuerdan con la bibliografía, por ser una muestra tan pequeña y sólo a la mitad de ésta se le realizaron los test.

Se define rehabilitación pulmonar como un programa de ejercicios de entrenamiento $\mathrm{y}$ al menos uno de los siguientes componentes: educación, apoyo psicosocial y/o nutricional, como ha sido definido en otros estudios ${ }^{(13,14)}$.

El GOLD (Global Initiative for Chronic Obstructive Lung Disease) documenta evidencia A en la mejoría de la disnea, estado de salud y tolerancia al ejercicio en paciente estables; y evidencia $\mathrm{B}$ en la disminución de las hospitalizaciones, posterior a un programa de rehabilitación pulmonar ${ }^{(15)}$.

Las guías prácticas clínicas de la ACCP/AACVPR, basadas en la evidencia, brindan recomendaciones para la rehabilitación pulmonar, que se irán describiendo a continuación según los objetivos e incluyendo otros estudios para reforzar la discusión de los resultados obtenidos.

Basados en la escala de disnea mMRC, Según la literatura, la disnea es uno de los factores que poseen mayor repuesta a los programas de rehabilitación. Se reporta como evidencia $1 \mathrm{~A}$ la mejoría de la disnea. En revisiones previas, se había descrito como nivel $\mathrm{B}$ de evidencia apoyado por la recomendación que mejora la calidad de vida relacionada con la salud, pero publicaciones adicionales más recientes refuerzan esta conclusión $\mathrm{y}$ actualizan la evidencia a nivel $\mathrm{A}^{(14)}$.

En los últimos años, se ha acumulado gran evidencia acerca de la mejoría que la rehabilitación respiratoria produce en la disnea y en la capacidad de ejercicio, principalmente en los pacientes con EPOC. Los resultados de un metaanálisis en 1996, con 14 ensayos controlados, pusieron de manifiesto una mejoría clínicamente importante en las dimensiones de disnea y control sobre la enfermedad del CRQ
(Chronic Respiratory Disease Questionnaire). Posteriormente, un buen número de estudios han confirmado el beneficio de ésta en pacientes con enfermedades pulmonares crónicas, inclusive las restrictivas. Además, se reporta que éste efecto positivo parece perdurar a largo plazo ${ }^{(16)}$.

Los datos funcionales después del entrenamiento coinciden con estudios previos que muestran una mejoría en la caminata de seis minutos $(\mathrm{p}=$ 0,009, Tabla II). Sin embargo, algunos resultados podrían verse condicionados al ser una muestra tan pequeña. De los 12 pacientes incluidos, sólo 9 concluyeron el programa con todos los parámetros, dentro de éstos, la C6M. De los 9 pacientes, sólo 2 poseían el diagnóstico de EPID, cinco de EPOC y dos de asma, con mejoría promedio de la caminata de seis minutos de 65,5 $\mathrm{m}, 33,92 \mathrm{~m}$ y $25,5 \mathrm{~m}$, respectivamente.

Di Meo y colaboradores, en un estudio realizado en 74 adultos mayores, con una media de edad de 74,2 años, documentó una mejoría significativa de la C6M (mayor de 54 metros) en el 50\% de la muestra y un promedio de mejoría de 76,2 m (DS $65 \mathrm{~m}$, rango -45 a $270 \mathrm{~m}$ ) de pacientes con $\mathrm{EPOC}^{(17)}$.

En otro estudio realizado por Salhi y colaboradores en 29 pacientes con enfermedades restrictivas, tomando los mismos cortes de diferencia mínima significativa de $54 \mathrm{~m}$, se encontró un promedio de mejoría de $64+-67 \mathrm{~m}$ a las 12 semanas de terapia y de $81+-84 \mathrm{~m}$ después de 24 semanas, y sólo 34\% mejoraron más de 54 metros a las doce semanas y 50\% a las 24 semanas ${ }^{(18)}$. La diferencia significativa en el actual estudio, se debe a que es una muestra muy pequeña y se incluyen neumopatías crónicas obstructivas y restrictivas con patrones demográficos diferentes.

No hay asociación entre la mejoría y los índices de función pulmonar, tanto en los adultos mayores como en la población más joven ${ }^{(17)}$.

El número de comorbilidades, la severidad de la enfermedad pulmonar, el grado de hipoxemia y el número de exacerbaciones determinan la respuesta a un programa de rehabilitación pulmonar, según un estudio realizado en el 2012, en 320 pacientes con EPOC. Además, indican no correlación significativa con la edad y género, lo que coincide con los resultados actuales ${ }^{(19)}$. Los 
efectos de la rehabilitación pulmonar en cuanto a la tolerancia al ejercicio son independientes a la edad, inclusive en los mayores de 80 años ${ }^{(17)}$.

El papel potencial de la debilidad muscular ventilatoria y posible fatiga, como causa de limitación ventilatoria y fallo respiratorio en pacientes con enfermedad respiratoria crónica, ha estimulado la introducción de su entrenamiento en los programas de rehabilitación. Varios estudios sobre el entrenamiento muscular inspiratorio han demostrados pequeñas diferencias en los parámetros fisiológicos y sintomáticos $^{(20,21,23)}$.

Los resultados en ocasiones han sido contradictorios y dependen de la modalidad de la resistencia aplicada y de la intensidad de ésta; aún así, se señala que el entrenamiento de resistencia con patrón respiratoria controlado y generación de presión sustancial durante la inspiración puede dar lugar a apreciables mejorías de las fuerzas, resistencia y sensación de disnea, con la consiguiente reducción de la incomodidad de las actividades de la vida diaria, aumento de la capacidad funcional y mejoría de la calidad de vida ${ }^{(21)}$.

En el estudio, los pacientes obtuvieron una mejoría significativa de la PIMáx en el $83 \%$, posterior al proceso de rehabilitación. Este resultado es coherente con lo demostrado por otros estudios, como el realizado por Larson et al, que fue el primero en demostrar un aumento estadísticamente significativo de la fuerza muscular inspiratoria, resistencia muscular respiratoria y tolerancia al ejercicio en pacientes con EPOC, moderada o grave. Posteriormente, Belman y Shadmehr demostraron una mejoría significativa de la fuerza muscular inspiratoria en un grupo de pacientes con EPOC, en los que se compararon los ejercicios de alta resistencia con lo de baja resistencia, aplicada durante 15 minutos diarios por seis semanas.

En otro estudio aleatorizado realizado en 35 pacientes, por Serón et al, en el que al grupo control se le administró un entrenamiento con un $40 \%$ de resistencia y al grupo control con resistencia mínima, se demostró una mejoría de la PIMáx de $11,5 \mathrm{~cm} \mathrm{H} 2 \mathrm{O}$ a favor del grupo experimental en el primer mes $(p=0,02)$, sin encontrase diferencias significativas en otras variables como el FEV1 y la C6M (21). Al segundo mes de entrenamiento, no se encontraron diferencias significativas entre ambos grupos. Al igual que se ha reportado en otros estudios, probablemente el porcentaje de resistencia está asociado más al tiempo de respuesta que a la totalidad de la respuesta. Es necesario estudiar su efecto a largo plazo y su impacto sobre la calidad de vida en un mayor número de pacientes. Cabe agregar al análisis, que tanto este estudio como otros similares evalúan la fuerza muscular a través de la PIMáx, pero puede ser necesario emplear técnicas que midan directamente la contractilidad diafragmática, como la estimulación magnética del nervio frénico, o utilizar como medidas de impacto las basadas en aspectos clínicos o funcionales, como la que se pretendió en este trabajo al medir la calidad de vida relacionada con la salud ${ }^{(21)}$.

En cuanto a los parámetros de la espirometría, que también miden función pulmonar $\mathrm{y}$ severidad de la enfermedad, en nuestro estudio como en las revisiones anteriores y literatura, no hay mejoría en el FEV1 o FEV1/FVC ${ }^{(22,23)}$.

La resistencia muscular de extremidades superiores e inferiores aumentó, al documentarse un incremento en el número de repeticiones en ambas al finalizar el programa. Para los objetivos del trabajo, no se realizaron cruces entre variables, con el cuestionario de Saint George, ni con la disnea, por el gran fraccionamiento del reporte en los niveles del percentil de la repeticiones, tanto de miembros inferiores como superiores.

En un metaanálisis de Pan y colaboradores en el $2012^{(24)}$, donde se incluyeron sólo siete estudios de los 316 seleccionados inicialmente, se concluyó que el incluir ejercicio en las extremidades superiores sin apoyo podría mejorar la disnea y la fatiga de los miembros superiores durante las ABVD en los pacientes con EPOC. Sin embargo, son necesarios más estudios para determinar cuáles son las mediciones para examinar la respuesta clínica y para definir el mejor o más adecuado ejercicio de éstas ${ }^{(24)}$.

En cuanto al ejercicio de los miembros inferiores, se ha evidenciado que el ejercicio aeróbico de éstos es capaz de contrarrestar la 
atrofia de los músculos esqueléticos, restableciendo parcialmente el metabolismo muscular normal y su morfología. Conlleva a mejoría en los síntomas, calidad de vida y capacidad de ejercicio ${ }^{(25)}$. Se han realizado estudios para documentar los cambios en las características de los músculos periféricos posterior a un programa de rehabilitación en paciente neumópatas crónicos, principalmente con EPOC. Posterior a éste, las fibras musculares aumentan significativamente, independientemete de la severidad de la enfermedad ${ }^{(23)}$.

Las guías de la ACCP/AACVPR basadas en la evidencia recomiendan: a) Los ejercicios de extremidades inferiores de alta intensidad producen beneficios fisiológicos mayores que los de más baja intensidad (Recomendación 1B). Sin embargo, tanto los de alta como baja intensidad ofrecen beneficios clínicos para éstos pacientes (Recomendación 1A); b) Agregar ejercicios de resistencia a los programas de rehabilitación pulmonar aumenta la fuerza y la masa muscular (Recomendación 1A); c) Entrenamiento sin resistencia o sin apoyo de las extremidades superiores es beneficioso para los pacientes con EPOC y deberían ser incluidos en los programas de rehabilitación (Recomendación 1A).

El índice de BODE es el más representativo para clasificar la severidad de las enfermedades pulmonares crónicas y ha sido utilizado para reportar la respuesta posterior a un programa de rehabilitación pulmonar y como predictor de mortalidad. La respuesta positiva en su mayoría es atribuida a la mejoría en la disnea y en la capacidad para realizar ejercicio. Resultados similares fueron encontrados en este trabajo con una respuesta significativa en el índice de BODE en el $100 \%$ de los pacientes que completaron los diferentes componentes de la escala $(\mathrm{p}=0,015)$. En éste índice, al igual que se describe en otros estudios, la mejoría, se presento principalmente en los componentes de disnea $(p=0,007)$ y la C6M $(\mathrm{p}=0,009)$.

En un estudio realizado en 52 pacientes con EPOC entre los 49 a 82 años, posterior a un programa de rehabilitación de tres meses, hubo mejoría en el índice de BODE en 25 de ellos (Grupo A), los otros 27 (Grupo B) se mantuvieron sin cambios. En cuanto al cuestionario de Saint George, ambos grupos respondieron, con una mejoría significativamente mayor en el grupo A $(37+/-5$ a $24+/-4$, p menor 0,01 ) vrs. el grupo B (de $35+/-6$ a $29+/-3$, p menor a 0,05). Además, la disminución de 4 a más puntos en el cuestionario de Saint George representa una respuesta clínica importante en la mejoría de la calidad de vida, lo cual se observó en el $92 \%$ del grupo A y en el $70 \%$ del grupo B ${ }^{(26,27)}$. En el cuestionario de Saint George, el $75 \%$ de los paciente obtuvo una respuesta positiva al programa, de los cuales ocho de nueve pacientes que mejoraron, reportaron una disminución significativa del total del puntaje ${ }^{(28)}$.

Según Alonso y colaboradores, no se ha observado correlación entre el FEV1 y el cuestionario de Saint George. En el presente estudio, la relación entre el $\mathrm{FEV}_{1}$ y la calidad de vida reportó una gran variabilidad. Este resultado, que a primera vista parece contradictorio, no es infrecuente al revisar la bibliografía al respecto. En un estudio realizado por Osman et en 1997, se concluyó que no había relación entre la calidad de vida en su valor global y por escalas con valores espirométricos, pero sí con el número de ingresos hospitalarios. El estudio que realizaron Berry et al, en el que entrenaron a pacientes con EPOC, demostró que la afectación de la calidad de vida es independiente del grado de obstrucción bronquial (29).

En nuestro estudio, aunque débilmente, queda patente que un elevado valor del índice BODE repercute de forma negativa en la calidad de vida.

La actividad como subescala del SGRQ participa de forma decisiva en la correlación con el índice BODE. Al disminuir la actividad en estos pacientes, aumenta la morbimortalidad. Dentro de las subescalas del SGRQ, se observó una respuesta mayor posterior al programa de rehabilitación en la actividad y síntomas, independiente del grado de afectación ${ }^{(27)}$.

\section{CONCLUSIONES}

En el grupo de pacientes analizados, de los cuales la mayoría presentaban EPOC severo, seguidos por EPID y asma, se documenta la eficacia de un programa de rehabilitación pulmonar, con mejoría en el $42 \%$ de la disnea 
según la escala del mMRC, sin deterioro o sin cambios en el resto de la muestra.

Hubo una mejoría significativa en la caminata de seis minutos $(\mathrm{p}=0,009)$, la fuerza muscular de los músculos respiratorios $\mathrm{y}$ en la resistencia muscular de extremidades superiores $(\mathrm{p}=0,004)$ y inferiores $(p=0,007)$, siendo mayor en los hombres, menores de 80 años y FEV1 mayor de $80 \%$.

Con respecto a la calidad de vida relacionada con la salud, el cuestionario de Saint George mejoró en cada uno de sus componentes y su totalidad.

\section{BIBLIOGRAFÍA}

1. Fernández X. Primer Informe de la Persona Adulta Mayor en Costa Rica. Universidad de Costa Rica, Octubre 2007.

2. Divo M, y Pinto-Plata V. Role of Exercise in Testing and in Therapy of COPD. Medicine Clinic of North America 2012; 96: 753 - 766.

3. Halter J, Ouslander J, Tinetti $\mathrm{M}$ et al. Hazzard's Geriatric Medicine and Gerontology. Six Edition. McGraw Hill Medical 2009; pags $983-1007$.

4. Regiane V, Gorostiza A, Gáldiz JB, López E et al. Beneficios de un programa de rehabilitación respiratoria domiciliaria en pacientes con EPOC grave. Archivos de Bronconeumología 2007; 43: 599 - 604.

5. De Lucas P. ¿Cuánto debe durar la rehabilitación respiratoria?. Archivos de Bronconeumología 2001; 37: 459 - 460.

6. Rikli, R. y Jones, C. Functional fitness nor ma ti ve sco res for com mun nity-re si ding adults, ages 60-94. Journal of Aging and Physical Activity, 7 1999; 160-179.
La mayor respuesta se documentó en los síntomas, sin lograr documentarse una diferencia significativa.

En el índice de BODE se documentó una mejoría significativa posterior a la finalización del programa. En cuanto a sus componentes, la respuesta significativa se reportó en la caminata de seis minutos y en la disnea.

No se presentaron cambios en los parámetros espirométricos posterior al programa.

7. Rikli, R. y Jones, C. Senior fitness test manual. Estados Unidos de América. Human Kinetics 2001.

8. Bestall J C, Paul E A, Garrod R, Garnham R, Jones P W, Wedzicha J A. Usefulness of the Medical Research Council (MRC) dyspnoea scale as a measure of disability in patients with chronic obstructive pulmonary disease. Thorax 1999; 54: 581-6

9. Cote C G, Celli B R. Pulmonary rehabilitation and the BODE index in COPD. Eur Respir J 2005; 26: 630- 6.

10. Pathy J, Sinclair A, Morley J. Principles and Practice of Geriatric Medicine. Fourth Edition. Wiley Editorial, 2006; pags 665-730.

11. Connolly M, Shaw L. Respiratory Disease in Old Age. Research into Ageing Workshop. London, 1998. Age and Ageing 2000; 29: 281-285.

12. Matthews B. Alzheimer Disease Update. Continuum Lifelong Learning Neurol 2010;16(2):15-30.

13. Ries AL, Kaplan RM, Limberg TM, Prewitt LM. Effects of pulmonary rehabilitation on physiologic and psychosocial outcomes in patients with chronic obstructive pulmonary 
disease. Annals of Internal Medicine 1995; 122: 823 .

14. Ries AL, Baldoff GS, Carlin BW, et al. Pulmonary Rehabilitation: Joint

15. Di Meo F, Pedone C, Lubich S et al. Age does not hamper the response tp pulmonary rehabilitation of COPD patients. Age and Ageing 2008: 37 (5): 530 - 535 .

16. Salhi B, Troosters $\mathrm{T}$, Behaegel $\mathrm{M}$ et al. Effects of Pulmonary Rehabilitation in Patients with Restrictive Lung Diseases. Chest 2010; 137 (2): 273 - 279.

17. Nonna S. Factors Associated with Outcome among COPD Patients Undergoing Pulmonar Rehabilitation. Chest 2013; 144: 829A.

18. Tout R, Tavara L, Hamili M. The effects of respiratory nuscle training on improment of the internal and external thoraco - pulmonary respiratory mechanism in COPD paatients. Annals of Physical and Rehabilitation Medicine; 56 (3): 193 - 211.

19. Serón P, Riedemann $\mathrm{P}, \mathrm{Muñoz} \mathrm{S}$, et al. Efecto del entrenamiento muscular inspiratorio sobre la fuerza muscular y la calidad de vida en pacientes con limitación crónica del flujo aéreo. Ensayo Clínico aleatorizado. Archivos de Bronconeumología 2005; 41 (11): 601606.

20. Crisafulli E, Gorgone $\mathrm{P}$, Vagaggini $\mathrm{B}$, et al. Efficacy of standard rehabilitation in COPD outoatients with comorbidities. European Respiratory Journal 2010; 26: 1042.

21. Miranda G, Gómez A, Pleguezuelos E, Capellas L. Rehabilitación respiratoria en
ACCP/AACVPR Evidence - Based Clinical Practice Guidelines. Chest 2007; 131: 4S.

España. Estudio SORECAR. Rehabilitación 2011; 45 (3): 247 - 255.

22. Pan L, Guo YZ, Yan JH, et al. Does upper extremity exercise improve disnea in patients with COPD? A meta-analysis. Respiratory Medicine 2012; 106: 151

23. Costi S, Crisafulli E, Antoni FD, et al. Effects of unsupported upper extremity exercise training in patients with COPD: a randomized clinical trial. Chest 2009; 136: 387.

24. Kavoura P, Harikiopoulou M, Dumitru S, et al. Does pulmonary rehabilitation - induced improvement in BODE index correlate with improvements in anxiety and depression and quality of life?. CHEST 2009; 136: 52S.

25. Nasis OG, Vogiatzis I, Stratakos G et al. Effects of interval - load versus constant - load training on the BODE index in COPD patients. Respiratory Medicine 2009; 103: 1392.

26. American College of Chest Physicians (ACCP). ACCP Pulmonary Medicine Board Review. 25th Edition. American College of Chest Physicians. 2009; Pags: 113 - 118.

27. Rosenberg S, Kalhan R. An Integrated Approach to the Medical Treatment of Chronic Obstructive Pulmonary Disease. Medical Clinic of North America 2012; 96: 811-826.

\section{CONFLICTO DE INTERÉS Y/O AGRADECIMIENTOS}

Los autores declaran que no existió ningún conflicto de interés en el presente reporte. 


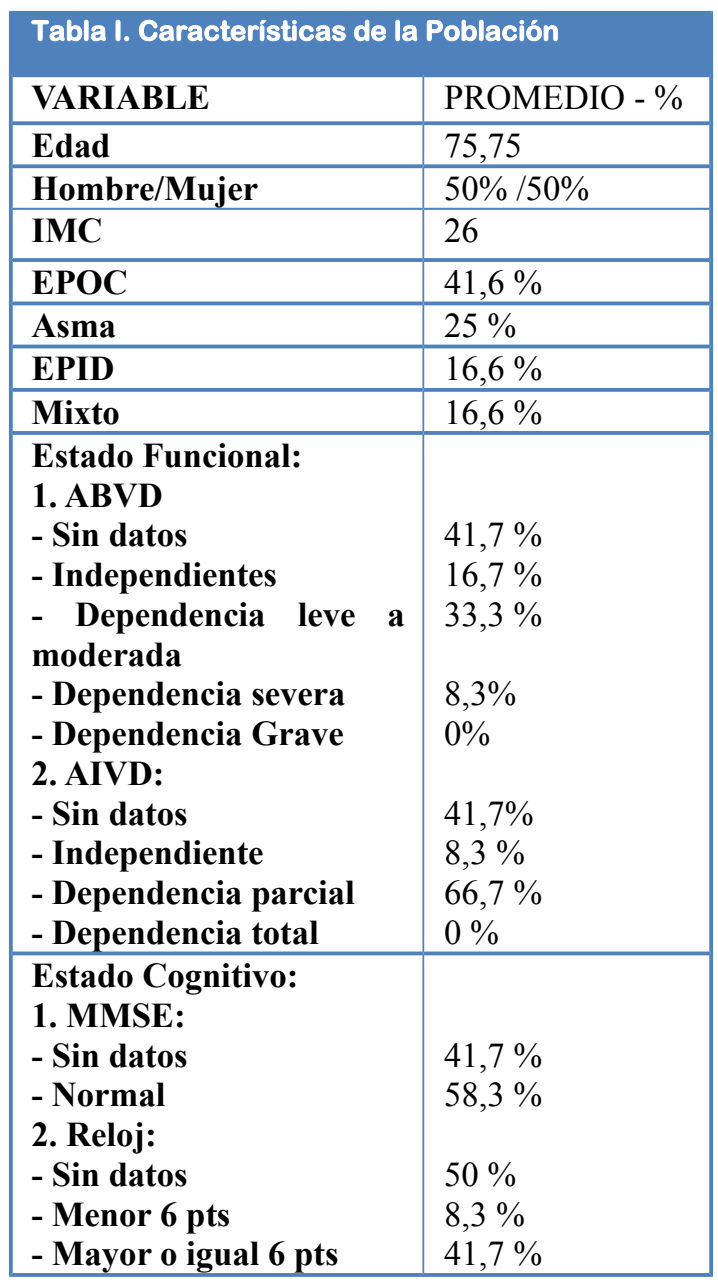

Fuente: HNGG 


\begin{tabular}{|c|c|c|c|c|c|c|}
\hline & $\mathbf{N}$ & Promedio & $\begin{array}{l}\text { Desviación es- } \\
\text { tándar }\end{array}$ & Mínimo & Máximo & Valor de $p$ \\
\hline C6M ING (mts) & 12 & 281 & 91,4 & 122 & 440 & \multirow{2}{*}{0,009} \\
\hline C6M EGR (mts) & 9 & 333 & 107,6 & 163 & 499 & \\
\hline C6M ING (\%) & 12 & 64,8 & 20,1 & 29 & 95 & \multirow[b]{2}{*}{0,008} \\
\hline C6M EGR (\%) & 9 & 75 & 18,7 & 42 & 104 & \\
\hline
\end{tabular}

Cuadro 2. Comparación de los valores promedio de la Prueba de Caminata de seis minutos al ingreso y al egreso del Programa de Rehabilitación Pulmonar en los pacientes del Hospital Nacional de Geriatría y Gerontología, de mayo del 2012 a julio del 2013

Fuente-. HNGG

\begin{tabular}{|c|c|c|c|c|}
\hline VARIABLE & $\begin{array}{c}\text { \% Promedio de } \\
\text { mejoría }\end{array}$ & Unidades & $\begin{array}{c}\text { TL de ptes con } \\
\text { mejoría }\end{array}$ & $\begin{array}{c}\% \text { de ptes con mejo- } \\
\text { ría }\end{array}$ \\
\hline CVF & 2,07 & $-0,025 \mathrm{~L}$ & 6 & 50 \\
\hline FEV1 & 0,01 & $4.16 \mathrm{~L}$ & 6 & 58,3 \\
\hline PIMáx & 0,93 & $16,6 \mathrm{kPa}$ & 11 & 91,7 \\
\hline PEMáx & 1,33 & $-0,33 \mathrm{kPa}$ & 6 & 50 \\
\hline
\end{tabular}

Cuadro 3. Comparación de los valores promedio de los volúmenes pulmonares y fuerza muscular respiratoria de los pacientes ingresados al Programa de Rehabilitación Pulmonar del Hospital Nacional de Geriatría y Gerontología, de mayo del 2012 a julio del 2013.

Fuente: HNGG 


\begin{tabular}{llll} 
(repeticiones) & $(+/-3,9)$ & $(+/-3,1)$ & \\
\hline RM MS & $10,5(+/-$ & 13,4 & 0,004 \\
(repeticiones) & $3,1)$ & $(+/-3,6)$ & \\
\hline BODE (pts) & 3,5 & 2,6 & 0,015 \\
\hline $\begin{array}{l}\text { Escala de } \\
\text { Disnea }\end{array}$ & 2,7 & 1,8 & 0.007 \\
MMRC & $(+/-1)$ & $(+/-1,4)$ & \\
\hline
\end{tabular}

Cuadro 4. Comparación de variables al ingreso y egreso del Programa de Rehabilitación Pulmonar del Hospital Nacional de Geriatría y Gerontología, de mayo del 2012 a julio del 2013.

Fuente: HNGG 